\title{
INFLUÊNCIA DA COLONIZAÇÃO MICORRÍZICA ARBUSCULAR SOBRE A NUTRIÇÃO DO QUIABEIRO'1
}

\author{
RICARDO LUÍS LOURO BERBARA ${ }^{2}$, LUÍS RODRIGUES FREIRE ${ }^{3}$ e MANLIO SILVESTRE FERNANDES ${ }^{4}$
}

\begin{abstract}
RESUMO - Foram estudados em casa de vegetação alguns parâmetros de crescimento em plantas de quiabo (Abelmoschus esculentus (L.) Moench cv. Piranema) colonizadas por dois grupos de fungos micorrízicos arbusculares, com o objetivo de determinar a influência dos inóculos na nutrição e morfologia radicular do quiabeiro. Um grupo continha apenas esporos de Acaulospora longula (A) enquanto o outro, esporos de oito espécies: Glomus occultum, Glomus aggregatum, Glomus microcarpum, Acaulospora longula, Acaulospora morrowae, Sclerocystis coremioides, Sclerocystis sinuosa, Scutellospora pellucida. As plantas foram submetidas a três níveis de P $\left(0,10\right.$ e $60 \mathrm{~kg} \mathrm{ha}^{-1} \mathrm{de}$ $\mathrm{P}_{2} \mathrm{O}_{5}$ ) e coletadas em três diferentes idades (22, 32 e 47 dias), com quatro repetições para cada tratamento. Foi determinado o acúmulo de $\mathrm{N}, \mathrm{P}, \mathrm{K}$, e Mg na raiz e parte aérea, bem como o influxo médio desses elementos e a área radicular. Os resultados indicaram, além da resposta positiva do quiabeiro ao $\mathrm{P}$, uma maior eficiência da inoculação com mistura de espécies apesar de o influxo médio, determinado aos 47 dias, apresentar maiores valores para o tratamento com A. longula.
\end{abstract}

Termos para indexação: micorriza, quiabo, nutrição de plantas, Abelmoschus esculentus.

\section{INFLUENCE OF ARBUSCULAR MYCORRHIZA FUNGI ON THE NUTRITION OF OKRA PLANT}

\begin{abstract}
An experiment was carried out in greenhouse to determine the influence of inoculation of two groups of arbuscular mycorrhizae on the nutrition and radicular morphology of the okra plant (Abelmoschus esculentus (L.) Moench cv. Piranema. One group had only Acaulospora longula spores and the other a spore mixture of eight species: Glomus occultum, Glomus aggregatum, Glomus microcarpum, Acaulospora longula, Acaulospora morrowae, Sclerocystis coremioides, Sclerocystis sinuosa, Scutellospora pellucida. The experiment was held in greenhouse conditions with three levels of $\mathrm{P}\left(0,10\right.$ and $60 \mathrm{~kg} \mathrm{ha}^{-1}$ of $\left.\mathrm{P}_{2} \mathrm{O}_{5}\right)$, three samplings dates (22, 32 and 47 days) and four replications. The accumulation of $\mathrm{N}, \mathrm{P}, \mathrm{K}, \mathrm{Ca}$ and $\mathrm{Mg}$ in roots and shoots, root area and their influx ratio were determined. The results made evident that the mixture of species was more efficient although there were higher ratios of influx with Acaulospora longula between 0 and $47^{\text {th. }}$ day.
\end{abstract}

Index terms: mycorrhiza, okra, plant nutrition, Abelmoschus esculentus.

\section{INTRODUÇÃO}

O maior crescimento de plantas causado por associações com fungos micorrízicos arbusculares (FMA) deve-se, principalmente, a sua melhor nutrição mineral. Esse efeito ocorre, em geral, pelo mai-

\footnotetext{
${ }^{1}$ Aceito para publicação em 27 de novembro de 1998.

${ }^{2}$ Eng. Agr., Ph.D., Prof. Adjunto, Universidade Federal Rural do Rio de Janeiro (UFRRJ), Dep. de Solos, CEP 23851-970 Seropédica, RJ.E-mail: berbara@ufrrj.br

${ }^{3}$ Eng. Agr., M.Sc., Prof. Titular, UFRRJ. E-mail: freire@ufrrj.br

${ }^{4}$ Eng. Agr., Ph.D., Prof. Adjunto, UFRRJ. E-mail: manlio@ufrrj.br
}

or volume de solo explorado por raízes micorrizadas. FMA diminuem a distância em que íons devem difundir-se pelo solo até serem absorvidos. Por esse motivo, o maior efeito da associação FMA será sobre elementos minerais que se movimentam por difusão e em plantas que se caracterizam por um sistema radicular rústico, com poucos pêlos radiculares ou ramificações (Baylis, 1975; Hayman et al., 1986). Íons que movimentamse por fluxo de massa têm sua absorção pouco afetada pela associação FMA (Harley \& Smith, 1983).

A nutrição mineral do quiabeiro caracteriza-se por uma extração de macros e micronutrientes lenta até os 20 dias, aumentando posteriormente (Costa et al., 1972). Segundo o autor, os teores de nutrien- 
tes nas folhas de plantas de quiabo sob estresse nutricional e coletadas aos 60 dias seriam próximos de $18,2 \mathrm{~g} \mathrm{~kg}^{-1}$ de N; $1,7 \mathrm{~g} \mathrm{~kg}^{-1}$ de P; $10,5 \mathrm{~g} \mathrm{~kg}^{-1}$ de $\mathrm{K} ; 29,4 \mathrm{~g} \mathrm{~kg}^{-1}$ de Ca e 2, $4 \mathrm{~g} \mathrm{~kg}^{-1}$ de $\mathrm{Mg}$, enquanto os desenvolvidos sem deficiências minerais em torno de 37,1 $\mathrm{g} \mathrm{kg}^{-1}$ de $\mathrm{N} ; 4,1 \mathrm{~g} \mathrm{~kg}^{-1}$ de P; $20 \mathrm{~g} \mathrm{~kg}^{-1}$ de $\mathrm{K} ; 37,3 \mathrm{~g} \mathrm{~kg}^{-1}$ de Ca e 8,6 $\mathrm{g} \mathrm{kg}^{-1}$ de Mg. Siemonsa (1982) concorda com tais valores à exceção de $\mathrm{Ca}$, considerado muito elevado. $\mathrm{O}$ autor indica para esse elemento concentrações em torno de 1,6\%. Asif \& Greig (1972), trabalhando com outras cultivares, indicaram para raízes de quiabo níveis de nutrientes de: $3,7 \mathrm{~g} \mathrm{~kg}^{-1}$ de N; $1,1 \mathrm{~g} \mathrm{~kg}^{-1}$ de P; 7,3 $\mathrm{g} \mathrm{kg}^{-1}$ de K ; $11,9 \mathrm{~g} \mathrm{~kg}^{-1} \mathrm{de}$ Ca e 2,3 $\mathrm{g} \mathrm{kg}^{-1} \mathrm{de} \mathrm{Mg}$. Já para as folhas adultas, os valores encontrados foram: $31,6 \mathrm{~g} \mathrm{~kg}^{-1}$ de N; 3, $\mathrm{g} \mathrm{kg}^{-1}$ de P; $15,4 \mathrm{~g} \mathrm{~kg}^{-1}$ de K; $23,2 \mathrm{~g} \mathrm{~kg}^{-1} \mathrm{de} \mathrm{Ca}$ e 2,2 $\mathrm{g} \mathrm{kg}^{-1}$ de Mg. Ahmad \& Tulloch (1969) acompanharam as variações com o tempo dos teores de $\mathrm{N}, \mathrm{P}, \mathrm{K}$ e $\mathrm{Mg}$ em plantas de quiabo submetidas a vários níveis de fertilidade. Os autores observaram uma queda geral desses valores com o tempo, à exceção do $\mathrm{P}$, que aumentava sua concentração nos tecidos até a oitava semana, caindo a seguir. O quiabo, por ser tradicionalmente cultivado no município de Itaguaí, RJ, por pequenos produtores, tem grande impacto econômico e social, sendo, portanto, pertinentes os estudos referentes às variações nos parâmetros fisiológicos, morfológicos e nutricionais dessa espécie vegetal quando colonizados por FMA por meio da determinação de alguns parâmetros de crescimento. Este trabalho teve como objetivo avaliar o efeito da inoculação com FMA e de doses de P sobre o desenvolvimento e influxo de nutrientes em plantas de quiabo em distintos estádios de crescimento.

\section{MATERIAL E MÉTODOS}

Esporos de FMA foram coletados, identificados e multiplicados, de dois solos representativos da região de Seropédica, município de Itaguaí, RJ (Séries Ecologia e Seropédica). As amostras de terra utilizadas no experimento em casa de vegetação foram coletadas de um Latossolo Vermelho-Amarelo série Piraí. Foi utilizada apenas a camada superficial do solo $(0-20 \mathrm{~cm})$ e a sua análise química revelou as seguintes características: $\mathrm{pH}$ água: 4,5; $\mathrm{Ca}^{2+}: 0,4 \mathrm{cmol}_{\mathrm{c}} \mathrm{kg}^{-1} ; \mathrm{Mg}^{2+}: 0,4 \mathrm{cmol}_{\mathrm{c}} \mathrm{kg}^{-1}$; $\mathrm{Na}^{+}: 21 \mathrm{mg} \mathrm{kg}^{-1} ; \mathrm{K}^{+}: 22 \mathrm{mg} \mathrm{kg}{ }^{-1} ; \mathrm{P}: 3 \mathrm{mg} \mathrm{kg}{ }^{-1} \mathrm{e}$
$\mathrm{Al}^{3+}: 0,5 \mathrm{cmol}_{\mathrm{c}} \mathrm{kg}^{-1}$. A análise granulométrica indicou: $50 \%$ de areia; $12 \%$ de silte; $38 \%$ de argila.

As amostras de terra foram secas ao ar, destorroadas, passadas em peneira de $2 \mathrm{~mm}$ e posteriormente autoclavadas $\left(120^{\circ} \mathrm{C}\right.$ por 2 horas). A terra fina secada ao ar (TFSA) foi então homogeneizada juntamente com o equivalente a $1.500 \mathrm{~kg} \mathrm{ha}^{-1}$ de calcário dolomítico e deixada em repouso por dois meses em vasos de 20 litros. Após essa fase, a terra foi novamente autoclavada e peneirada conforme descrito anteriormente. Vasos de plástico de 4,8 litros receberam então 4 kg de TFSA. Fertilizante fosfatado (superfosfato simples) foi aplicado quatro dias antes da semeadura com o equivalente às doses de 0 , 10 e $60 \mathrm{~kg} \mathrm{ha}^{-1}$ de $\mathrm{P}_{2} \mathrm{O}_{5}$. O fertilizante foi homogeneizado com a terra e, como procedimento comum, todos os vasos receberam na véspera da semeadura o equivalente a $90 \mathrm{~kg} \mathrm{ha}^{-1}$ de $\mathrm{K}_{2} \mathrm{O}$ na forma de cloreto de potássio, e $45 \mathrm{~kg} \mathrm{ha}^{-1}$ de $\mathrm{N}$ na forma de sulfato de amônio. Foram ainda aplicados $\mathrm{H}_{3} \mathrm{BO}_{3}: 2,86 \mathrm{~g} \mathrm{~L}^{-1} ; \mathrm{MnCl}_{2} \cdot \mathrm{H}_{2} \mathrm{O}: 1,81 \mathrm{~g} \mathrm{~L}^{-1}$; $\mathrm{ZnSO}_{4} \cdot 7 \mathrm{H}_{2} \mathrm{O}: 0,22 \mathrm{~g} \mathrm{~L}^{-1} ; \mathrm{CuSO}_{4} \cdot 5 \mathrm{H}_{2} \mathrm{O}: 0,08 \mathrm{~g} \mathrm{~L}^{-1} \mathrm{e}$ $\mathrm{H}_{2} \mathrm{MoO}_{4} \cdot \mathrm{H}_{2} \mathrm{O}: 0,02 \mathrm{~g} \mathrm{~L}^{-1}$. O Fe foi aplicado como citrato a partir de uma solução estoque de $1.000 \mathrm{mg} \mathrm{kg}^{-1} \mathrm{em}$ uma razão de $4 \mathrm{~mL} /$ vaso. Após a adubação, foram retiradas amostras de terra para análise e os resultados médios obtidos foram os seguintes: $\mathrm{pH}$ água $=5,8 ; \mathrm{Ca}^{2+}=1,5 \mathrm{cmol}_{\mathrm{c}} \mathrm{kg}^{-1}$; $\mathrm{Mg}^{2+}: 1,5 \mathrm{cmol}_{\mathrm{c}} \mathrm{kg}^{-1} ; \mathrm{Na}^{+}: 18 \mathrm{mg} \mathrm{kg}{ }^{-1} ; \mathrm{K}^{+}: 99 \mathrm{mg} \mathrm{kg}^{-1}$; $\mathrm{Al}^{3+}: 0$. Os valores de $\mathrm{P}$ indicaram $3,4 \mathrm{e} 10 \mathrm{mg} \mathrm{kg}^{-1}$ para os tratamentos com 0,10 e $60 \mathrm{~kg} \mathrm{ha}^{-1} \mathrm{de}_{2} \mathrm{O}_{5}$ aplicados, respectivamente.

O tratamento com FMA compreendeu além da testemunha (T), que não recebeu inoculação, dois outros: mistura de espécies (M) com oito espécies nativas previamente identificadas (Glomus occultum, Glomus aggregatum, Glomus microcarpum, Acaulospora longula, Acaulospora morrowiae, Sclerocystis coremioides, Sclerocystis sinuosa, Scutellospora pellucida) e Acaulospora longula (A) com esporos de apenas Acaulospora longula. Esta espécie foi escolhida por ter sido a mais freqüentemente encontrada nas amostras de terra coletadas em solos representativos da região de Itaguaí. Os potes receberam logo após a aplicação do fertilizante fosfatado uma quantidade de terra inoculada com cerca de 2.800 esporos/vaso. Não se utilizou mistura de inóculos (hifas, raízes infectadas etc.) devido à dificuldade encontrada em homogeneizá-la quanto ao potencial infectivo, pela técnica do número mais provável (NMP) descrito por Porter (1979). Os tratamentos com mistura de espécies receberam também cerca de 2.800 esporos contendo aproximadamente 350 esporos de cada uma das oito espécies isoladas. Da mesma forma, todos os vasos receberam filtrados contendo a microflora presente nos vasos de multiplicação. 
Os esporos coletados dos vasos multiplicadores pela técnica de extração citada por Daniels \& Skipper (1982) foram novamente identificados para eliminação de propágulos considerados contaminantes. Os esporos, desinfetados por imersão em placa-de-petri contendo uma solução de $0,5 \%$ de hipoclorito de sódio, por dois minutos, foram armazenados em frascos com água deionizada por cinco dias em condição ambiente com cerca de 35 esporos $\mathrm{mL}^{-1}$. Os tratamentos com mistura de espécies receberam, após forte agitação, $10 \mathrm{~mL}$ do conteúdo dos frascos referentes a cada uma das oito espécies. A suspensão era aplicada aos vasos a uma profundidade de 4-5 cm com a ajuda de uma pipeta volumétrica, totalizando-se aproximadamente 2.800 esporos por vaso.

Doze sementes de quiabo (Abelmoschus esculentus (L.) Moench cv. Piranema) tratadas previamente com hipoclorito de sódio $2 \%$, por cinco minutos, foram colocadas em cada vaso a $2 \mathrm{~cm}$ de profundidade. A emergência das plântulas foi relativamente uniforme e 10 dias após o plantio foi feito um desbaste, deixando-se duas plantas por vaso. Os vasos foram dispostos de forma inteiramente casualizada, sendo mudados de posição uma vez por semana, com quatro repetições dispostas em esquema fatorial simples totalizando 81 vasos (3 épocas de coleta x 3 níveis de $\mathrm{P}$ x 3 inóculos $\mathrm{x}$ 4 repetições). Foram feitas três coletas: aos 22, 32 e 47 dias após o plantio (DAP). Nelas adotou-se o mesmo procedimento para todos os tratamentos. As plantas eram cuidadosamente retiradas dos vasos e separadas em raiz e parte aérea. Imediatamente após a coleta era determinada a área radicular pelo método fotoelétrico (LI-CORLTD modelo 3.000) conforme descrito por Rossiello et al. (1995). Subamostras de raiz e parte aérea foram separadas para as determinações de N-total, P-total, $\mathrm{K}^{+}, \mathrm{Ca}^{2+}$ e $\mathrm{Mg}^{2+}$ após digestão com $\mathrm{H}_{2} \mathrm{O}_{2}+\mathrm{H}_{2} \mathrm{SO}_{4}$, conforme metodologia descrita por Tedesco (1982). As determinações de $\mathrm{Ca}^{2+} \mathrm{e} \mathrm{Mg}^{2+}$ foram realizadas por espectrofotometria de absorção atômica na Embrapa-Centro Nacional de Pesquisa de Solos. Com base nos respectivos valores de acúmulo de nutrientes e de área radicular, foi calculado o influxo líquido médio de N, P, K, Ca e Mg nos três períodos considerados segundo a relação $\left(\mathrm{N}_{2}-\mathrm{N}_{1}\right)$. $\left(\mathrm{LnA}_{2}-\mathrm{LnA}_{1}\right) /\left(\mathrm{A}_{2}-\mathrm{A}_{1}\right)$. $\left(\mathrm{t}_{2}-\mathrm{t}_{1}\right)$ onde: $\mathrm{N}$ indica $\mathrm{o}$ acúmulo de um dado elemento mineral; $\mathrm{A}$ a área radicular; e T o intervalo de tempo entre as coletas. A colonização radicular foi determinada de acordo com Philips \& Hayman (1970). O influxo líquido relativo foi determinado dividindo-se os valores do influxo líquido médio para cada elemento considerado dos tratamentos com FMA, por aqueles obtidos nos tratamentos sem FMA.

\section{RESULTADOS E DISCUSSÃO}

Nos dados analisados por épocas na Tabela 1, observa-se que o peso de matéria seca total das plantas no tratamento com mistura de espécies, independentemente do nível de $\mathrm{P}$, foi maior que os de Acaulospora longula e testemunha. Aos 47 dias, os tratamentos com mistura de espécies e com A. longula foram significativamente superiores à testemunha em todos os níveis de $\mathrm{P}$, quando considerado a planta inteira. Aos 22 dias, tal variação não foi detectada. Os resultados indicam que, independentemente do nível de $\mathrm{P}$, o tratamento com a mistura foi significativamente superior a $A$. longula e este à testemunha, com exceção da coleta aos 22 DAP. As observações na raiz e parte aérea apontam, em geral, para um mesmo padrão de comportamento. Os valores encontrados no nível $60 \mathrm{P}_{2} \mathrm{O}_{5}$ indicam uma diminuição da diferença entre os tratamentos com mistura de espécies e $A$. longula nos estádios mais avançados de desenvolvimento do quiabo. Observa-se também, nos três níveis de $\mathrm{P}$ e com a evolução do tempo, um distanciamento progressivo da produção de matéria seca das plantas inoculadas em relação às não inoculadas, indicando que a resposta à inoculação se expressa após 22 DAP. Os resultados de mistura de espécies nativas mostraram-se mais eficientes que $A$. longula isoladamente nas condições experimentais nos períodos anteriores a 47 DAP quando o peso da matéria seca total de $\mathrm{A}$ e $\mathrm{M}$ não diferiram estatisticamente (Tabela 1).

As respostas do quiabeiro à aplicação de $\mathrm{P}$, independentemente da inoculação com fungos micorrízicos, sugerem uma tendência geral de os valores de peso de matéria seca total serem significativamente maiores para o nível $60 \mathrm{~kg} \mathrm{ha}^{-1}$, seguido de $10 \mathrm{~kg} \mathrm{ha}^{-1} \mathrm{e}$ zero de $\mathrm{P}$ aplicado, à exceção da coleta realizada aos 22 dias na qual não se observaram variações significativas entre os níveis 0 e $10 \mathrm{~kg} \mathrm{ha}^{-1}$ de $\mathrm{P}_{2} \mathrm{O}_{5}$ (Tabela 1). Esses resultados sugerem que, quando observada a planta inteira, a resposta do quiabeiro à aplicação de $\mathrm{P}$ varia em função da dose de P aplicado.

Nos resultados de área radicular analisados por épocas (Tabela 2), observa-se que o tratamento com mistura de espécies (M) apresentou, em geral, va- 





lores maiores que os de $A$. longula (A), e este que os da testemunha (T). Os resultados de peso e área radicular sugerem que a mistura de espécies (M) é mais eficiente que $A$. longula isoladamente.

Estudos relativos à dinâmica de absorção iônica do quiabeiro (cultivares Santa Cruz e Piranema) não foram encontrados na literatura pesquisada. Portanto, como as referências obtidas a partir de observações com cultivares exóticas apenas indicam padrões gerais, elas não devem ser diretamente correlacionadas com os obtidos no presente estudo. Apesar desta ressalva, os valores obtidos neste trabalho encontram-se próximos aos limites estabelecidos (Ahmad \& Tulloch, 1969; Asif \& Greig, 1972; Krishna \& Bagyaraj, 1982). Segundo Siemonsa (1982), o N é o elemento mais extraído pela cultura do quiabo, seguido por $\mathrm{Ca}, \mathrm{K}, \mathrm{Mg}$ e $\mathrm{P}$. Costa et al. (1972) observaram um maior acúmulo de K seguido por $\mathrm{N}, \mathrm{Ca}, \mathrm{Mg}$ e $\mathrm{P}$. O presente trabalho indica valores coerentes com os apresentados por Siemonsa (1982) com a única exceção do Ca, que foi menos extraído que o K (Tabelas 3 e 4).
Os resultados de influxo líquido médio de nutrientes, parâmetro que indica a eficiência da raiz em absorver elementos minerais, sugerem a ocorrência de um mesmo padrão de comportamento para todos os íons estudados (Tabelas 3 e 4). Tal parâmetro é definido como o quanto de nutriente é absorvido por unidade da área radicular em função do tempo. Assim, era de se esperar que os tratamentos com inoculação ( $\mathrm{M} \mathrm{e} \mathrm{A}$ ) apresentassem os maiores valores. De forma geral, tal fato é observado em todos os tratamentos. Nota-se uma baixa eficiência de Acaulospora longula na absorção de nutrientes até os 32 dias. Na coleta realizada aos 47 DAP, entretanto, observam-se aumentos de influxo para $\mathrm{A}$ em relação a $\mathrm{M} \mathrm{e} \mathrm{T}$ (Tabela 5). A eficiência do FMA pode depender de sua capacidade de emitir hifas extra-radiculares (Bowen, 1987; Evans \& Miller, 1988; Sylvia, 1988). Hepper (1981) indica uma relação de 1:1 entre a biomassa intra e extra-radicular enquanto Sanders \& Sheikh (1983) encontraram taxas de crescimento de 0,4 e $0,7 \mathrm{~mm} \mathrm{dia}^{-1}$ para hifas internas e externas à raiz,

TABELA 2. Efeito da inoculação de fungos micorrízicos arbusculares, do nível de $P$ e da época de coleta sobre a área radicular $\left(\mathrm{cm}^{2}\right)$ em plantas de quiabo ${ }^{1}$.

\begin{tabular}{cccccc}
\hline Dias após o plantio & $\begin{array}{c}\text { Nível de } \mathrm{P}_{2} \mathrm{O}_{5} \\
\left(\mathrm{~kg} \mathrm{ha}^{-1}\right)\end{array}$ & $\mathrm{T}$ & $\mathrm{A}$ & $\mathrm{M}$ & Média \\
\hline 0 & 0 & $30,27 \mathrm{Ba}$ & $32,95 \mathrm{Ba}$ & $38,21 \mathrm{Ca}$ & $33,81 \mathrm{~B}$ \\
& 10 & $38,98 \mathrm{Bb}$ & $41,96 \mathrm{Bb}$ & $47,83 \mathrm{ABa}$ & $42,92 \mathrm{~B}$ \\
& 60 & $64,30 \mathrm{Aa}$ & $69,05 \mathrm{Aa}$ & $71,79 \mathrm{Ab}$ & $68,38 \mathrm{~A}$ \\
& Média & $44,52 \mathrm{a}$ & $47,99 \mathrm{a}$ & $52,61 \mathrm{a}$ & --- \\
\hline 0 & $43,69 \mathrm{Cb}$ & $63,10 \mathrm{Ca}$ & $79,70 \mathrm{Ca}$ & $62,16 \mathrm{C}$ \\
& 10 & $64,62 \mathrm{Bc}$ & $91,57 \mathrm{Cb}$ & $107,49 \mathrm{Ba}$ & $87,89 \mathrm{~B}$ \\
& 60 & $148,12 \mathrm{Ac}$ & $205,37 \mathrm{Ab}$ & $244,88 \mathrm{Aa}$ & $199,46 \mathrm{~A}$ \\
& Média & $85,48 \mathrm{c}$ & $120,01 \mathrm{~b}$ & $144,02 \mathrm{a}$ & --- \\
\hline 0 & $65,27 \mathrm{Cc}$ & $116,31 \mathrm{Cb}$ & $147,26 \mathrm{Ca}$ & $109,61 \mathrm{C}$ \\
& 10 & $114,48 \mathrm{Bb}$ & $203,27 \mathrm{Ba}$ & $244,04 \mathrm{BCa}$ & $187,26 \mathrm{BC}$ \\
& 60 & $231,63 \mathrm{Ac}$ & $374,77 \mathrm{Ab}$ & $437,93 \mathrm{Aa}$ & $348,11 \mathrm{~A}$ \\
& Média & $137,13 \mathrm{~b}$ & $231,45 \mathrm{ab}$ & $276,41 \mathrm{a}$ & --- \\
\hline
\end{tabular}

1 T: testemunha; A: inoculação com Acaulospora longula; M: inoculação com mistura de espécies; valores seguidos da mesma letra, minúscula nas linhas e maiúsculas nas colunas, não diferem significativamente entre si, pelo teste de Duncan (5\%), no mesmo período de amostragem. 


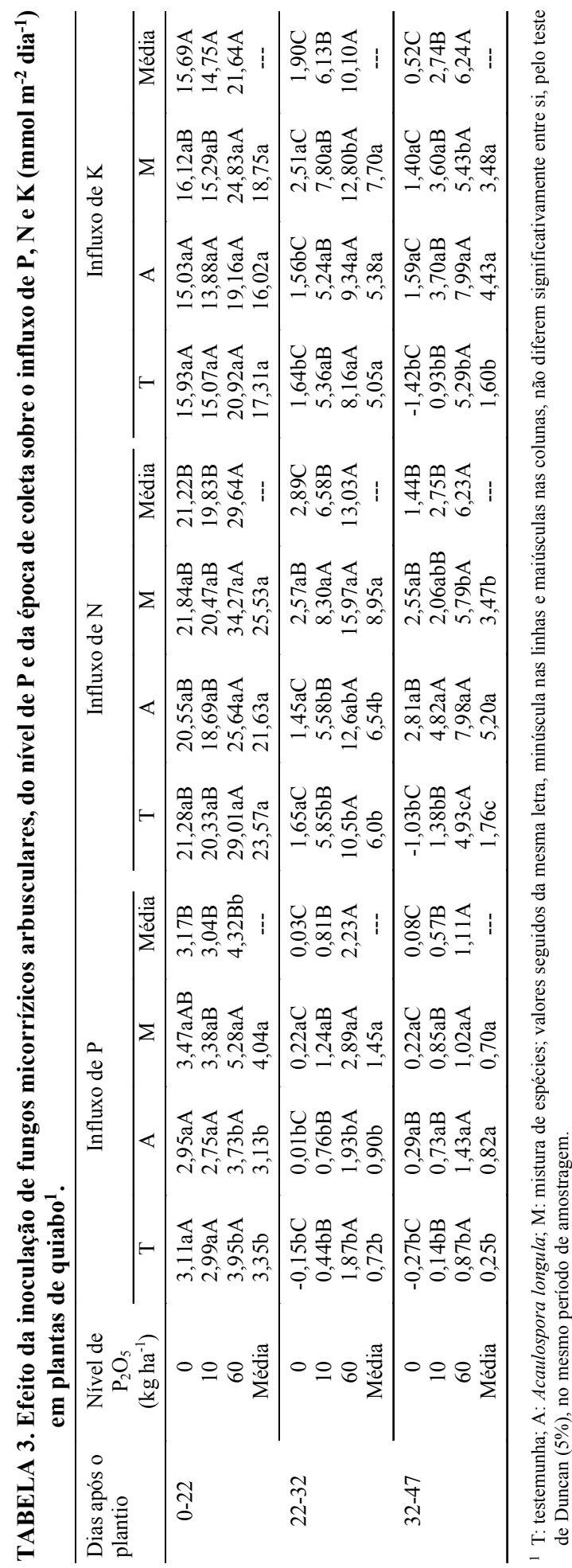

respectivamente. Bethlenfalvay et al. (1982) trabalhando com soja e Glomus mosseae sugerem que a relação entre a biomassa extra e intra-radicular varia de 7:1 para $0,4: 1$ entre a $4^{\mathrm{a}}$ e a $14^{\mathrm{a}}$ semanas respectivamente. Poder-se-ía propor que A. longula não seja uma espécie intrinsecamente eficiente no processo de absorção, translocação ou liberação de elementos minerais para o hospedeiro (Allsopp \& Stock, 1992; Smith \& Read, 1997). Entretanto, essa característica, que é fruto de uma interação genética, dificilmente se modificaria com o tempo conforme ocorreu no presente estudo.

De acordo com a Tabela 5, que indica para cada elemento a importância relativa do fungo micorrízico no influxo líquido médio de elementos ao longo do tempo, nota-se que $A$. longula poderia estar impondo à planta nas fases iniciais de seu desenvolvimento um dreno de fotoassimilados alto em relação ao do tratamento com mistura de espécies. As plantas podem, temporária ou permanentemente, apresentar respostas negativas quando micorrizadas (Smith $\&$ Read, 1997). Esse fenômeno pode ser observado no tratamento com A. longula, época I (Tabela 5). Uma redução temporária pode ocorrer quando na sua fase inicial de crescimento a planta desloca quantidades relativamente altas de fotoassimilados ao simbionte, e este ainda não absorve ou transloca quantidades suficientes de nutrientes (Bonfante \& Perotto, 1995). Os resultados indicam um maior impacto dos FMA no influxo líquido médio ao longo do período experimental, expressando a necessidade do estabelecimento da simbiose com a emissão de hifas intra e extra-radiculares (Tabela 5).

Os maiores valores de influxo líquido médio obtidos no período do 0-22 dias, e com os maiores níveis de $\mathrm{P}$, eram esperados, pois são nessas condições que o influxo é máximo (Pacovsky \& Fuller, 1986). Em geral, as maiores variações entre os tratamentos com e sem FMA ocorreram no influxo de P. Tal observação é coerente com o fato de que as maiores respostas à presença de FMA ocorrerem com os elementos pouco móveis no solo, como $\mathrm{P}$, e as menores para os que se movimentam por fluxo de massa, como o N (Bolan, 1991), o que explica as menores variações em relação a $\mathrm{T}$ conforme discutem Smith \& Gianinazzi-Pearson (1988). Os maiores valores absolutos de influxo, entretanto, foram 
TABELA 4. Efeito da inoculação de fungos micorrízicos arbusculares, do nível de P, e da época de coleta sobre o influxo de $\mathrm{Ca}$ e $\mathrm{Mg}\left(\mathrm{mmol} \mathrm{m}^{-2} \mathrm{dia}^{-1}\right)$ em plantas de quiabo ${ }^{1}$.

\begin{tabular}{|c|c|c|c|c|c|c|c|c|c|}
\hline \multirow{2}{*}{$\begin{array}{l}\text { Dias após } \\
\text { o plantio }\end{array}$} & \multirow{2}{*}{$\begin{array}{l}\text { Nível de } \\
\mathrm{P}_{2} \mathrm{O}_{5} \\
(\mathrm{~kg} / \mathrm{ha})\end{array}$} & \multicolumn{4}{|c|}{ Influxo de $\mathrm{Ca}$} & \multicolumn{4}{|c|}{ Influxo de $\mathrm{Mg}$} \\
\hline & & $\mathrm{T}$ & $\mathrm{A}$ & $\mathrm{M}$ & Média & $\mathrm{T}$ & $\mathrm{A}$ & $\mathrm{M}$ & Média \\
\hline \multirow{4}{*}{$0-22$} & 0 & $9,48 \mathrm{aA}$ & $8,86 \mathrm{aA}$ & $9,75 \mathrm{aA}$ & $9,36 \mathrm{~A}$ & $7,75 \mathrm{aA}$ & $7,70 \mathrm{aA}$ & $8,19 \mathrm{aA}$ & $7,88 \mathrm{~A}$ \\
\hline & 10 & $10,34 \mathrm{aA}$ & $9,52 \mathrm{aA}$ & $10,59 \mathrm{aA}$ & $10,15 \mathrm{~A}$ & $7,30 \mathrm{aA}$ & $6,53 \mathrm{aA}$ & $7,53 \mathrm{aA}$ & $7,21 \mathrm{~A}$ \\
\hline & 60 & $16,34 \mathrm{aB}$ & $14,99 \mathrm{aB}$ & $19,60 \mathrm{aB}$ & $16,98 \mathrm{~B}$ & $11,69 \mathrm{aA}$ & $10,56 \mathrm{aA}$ & $14,00 \mathrm{aB}$ & $12,08 \mathrm{~B}$ \\
\hline & Média & $12,05 \mathrm{a}$ & $11,12 \mathrm{a}$ & $13,31 \mathrm{a}$ & --- & $8,91 \mathrm{a}$ & $8,26 \mathrm{a}$ & $9,91 \mathrm{a}$ & --- \\
\hline \multirow[t]{4}{*}{$22-32$} & 0 & $0,77 \mathrm{aA}$ & $0,72 \mathrm{aA}$ & $1,23 \mathrm{bA}$ & $0,91 \mathrm{~A}$ & $1,03 \mathrm{aA}$ & $0,60 \mathrm{aA}$ & $1,16 \mathrm{bA}$ & $0,93 \mathrm{~A}$ \\
\hline & 10 & $2,75 \mathrm{aB}$ & $2,87 \mathrm{aB}$ & $4,38 \mathrm{bB}$ & $3,33 \mathrm{~B}$ & $2,94 \mathrm{aA}$ & $2,89 \mathrm{aB}$ & $4,17 \mathrm{bB}$ & $3,33 \mathrm{~B}$ \\
\hline & 60 & $6,01 \mathrm{aC}$ & $7,21 \mathrm{aC}$ & $10,00 \mathrm{aC}$ & $7,74 \mathrm{C}$ & $6,08 \mathrm{aB}$ & $6,95 \mathrm{aC}$ & $9,34 \mathrm{aC}$ & $7,46 \mathrm{C}$ \\
\hline & Média & $3,18 \mathrm{a}$ & $3,60 \mathrm{a}$ & $5,20 \mathrm{a}$ & --- & $3,35 \mathrm{a}$ & $3,48 \mathrm{a}$ & $4,98 \mathrm{a}$ & --- \\
\hline \multirow[t]{4}{*}{$32-47$} & 0 & $-0,74 \mathrm{aA}$ & $0,92 \mathrm{bA}$ & $0,85 \mathrm{bA}$ & $0,34 \mathrm{~A}$ & $-0,96 \mathrm{aA}$ & $0,71 \mathrm{bA}$ & $0,62 \mathrm{bA}$ & $0,12 \mathrm{~A}$ \\
\hline & 10 & $0,74 \mathrm{aB}$ & $1,94 \mathrm{aB}$ & $2,71 \mathrm{bB}$ & $1,80 \mathrm{~B}$ & $0,62 \mathrm{aB}$ & $1,77 \mathrm{bB}$ & $2,34 \mathrm{bB}$ & $1,58 \mathrm{~B}$ \\
\hline & 60 & $2,93 \mathrm{aC}$ & $4,86 \mathrm{bC}$ & $3,27 b \mathrm{c}$ & $3,69 \mathrm{C}$ & $2,27 \mathrm{aC}$ & $4,10 \mathrm{bC}$ & $2,66 \mathrm{aB}$ & $3,01 \mathrm{C}$ \\
\hline & Média & $0,98 \mathrm{a}$ & $2,57 \mathrm{~b}$ & $2,28 b$ & --- & $0,64 a$ & $2,19 b$ & $1,87 b$ & --- \\
\hline
\end{tabular}

1 T: testemunha; A: Acaulospora longula; M: mistura de espécies; valores seguidos da mesma letra, minúscula nas linhas e maiúsculas nas colunas, não diferem significativamente entre si, pelo teste de Duncan (5\%), no mesmo período de amostragem.

TABELA 5. Relação entre o influxo líquido médio de $\mathrm{P}, \mathrm{N}, \mathrm{K}, \mathrm{Ca}$ e Mg em plantas de quiabo com e sem colonização micorrízica.

\begin{tabular}{cccc}
\hline Dias após & Elemento & \multicolumn{2}{c}{ Influxo relativo $^{1}$} \\
\cline { 3 - 4 } o plantio & & $\mathrm{A} / \mathrm{T}$ & $\mathrm{M} / \mathrm{T}$ \\
\hline $0-22$ & $\mathrm{P}$ & 0,93 & 1,21 \\
& $\mathrm{~N}$ & 0,92 & 1,08 \\
& $\mathrm{~K}$ & 0,92 & 1,08 \\
& $\mathrm{Ca}$ & 0,91 & 1,10 \\
& $\mathrm{Mg}$ & 0,92 & 1,10 \\
\cline { 2 - 4 } $22-32$ & $\mathrm{P}$ & 1,25 & 2,00 \\
& $\mathrm{~N}$ & 1,09 & 1,49 \\
& $\mathrm{~K}$ & 1,06 & 1,52 \\
& $\mathrm{Ca}$ & 1,13 & 1,60 \\
$32-47$ & $\mathrm{Mg}$ & 1,03 & 1,48 \\
\cline { 2 - 4 } & $\mathrm{P}$ & 3,28 & 2,80 \\
& $\mathrm{~N}$ & 2,95 & 1,97 \\
& $\mathrm{~K}$ & 2,76 & 2,10 \\
& $\mathrm{Ca}$ & 2,60 & 2,32 \\
& $\mathrm{Mg}$ & 3,42 & 2,92 \\
\hline
\end{tabular}

1 A/T: valores médios de influxo de Acaulospora longula dividido pelos da testemunha, sem inoculação; M/T: valores médios de influxo de mistura de oito espécies de fungos micorrízicos arbusculares dividido pelos valores da testemunha sem inoculação. em relação a $\mathrm{N}$, seguido de $\mathrm{K}, \mathrm{Ca}, \mathrm{Mg}$ e $\mathrm{P}$. Os valores negativos de influxo do tratamento $\mathrm{T}$, obtidos para todos os elementos minerais no nível $0 \mathrm{P}$ (47 DAP), e apenas para $\mathrm{P}$ aos $32 \mathrm{DAP}$, sugerem que a planta, nessas fases, está perdendo elementos minerais para o ambiente mais do que está absorvendo. Tal fato já foi observado em outros trabalhos (Jakobsen, 1986) e indicam momentos em que a presença da associação micorrízica é mais importante.

\section{CONCLUSÕES}

1. O influxo de nutrientes e a matéria seca são, em geral, superiores no tratamento com mistura de espécies de fungos micorrízicos; o tratamento com Acaulospora longula é superior à testemunha a partir do $32^{\circ}$ dia após o plantio.

2. A área radicular é maior nos tratamentos com fungos micorrízicos arbusculares, em especial no de mistura de espécies, a partir do $32^{\circ}$ dia após o plantio. 
3. Os tratamentos com mistura de espécies indicam maiores valores de influxo de nutrientes, e Acaulospora longula destaca-se da testemunha apenas na coleta feita 47 dias após o plantio.

4. Os valores de matéria seca, influxo e área radicular são, em geral, superiores nos tratamentos com $60 \mathrm{~kg} \mathrm{ha}^{-1}$ de $\mathrm{P}$.

\section{REFERÊNCIAS}

AHMAD, N.; TULLOCH, L.I. Effect of nitrogen, phosphorus, potassium and magnesium on yield and nutrient content of okra (Hibiscus esculentus L.). Agronomy Journal, v.60, p.353-356, 1969.

ALLSOPP, N.; STOCK,W.D. Mycorrhizas, seed size and seedling establishment in a low nutrient environment. In: READ, D.H.; FITTER, A.H.; ALEXANDER, I.J. (Eds.). Mycorrhizas in Ecosystems. Wallingford: CAB International, 1992. p.59-64.

ASIF, M.I.; GREIG, J.K. Effects of N, P and K fertilization on fruit yield, macro and micronutrient level and nitrate accumulation in okra (Abelmoschus esculentus (L.) Moench). Journal of the American Society for Horticultural Science, v.97, n.4, p.440442, 1972.

BAYLIS, G.T.S. The magnolioid mycorrhiza and mycotrophy in the root systems serived from it. In: SANDERS, F.E.; MOSSE, B.; TINKER, P.B. (Eds.). Endomycorrhizas. London: Academic, 1975. p.373-389.

BETHLENFALVAY, G.J.; PACOVSKY, R.S.; BROWN, M.S.; FULLER, G. Mycotrophic growth and mutualistic development of host plant and fungal endophyte in an endomycorrhizal symbiosis. Plant and Soil, Netherlands, v.68, p.43-54, 1982.

BOLAN, N.S. A critical review on the role of mycorrhizal fungi in the uptake of phosphorus by plants. Plant and Soil, Netherlands, v.134, p.189-207, 1991.

BONFANTE, P.; PEROTTO, S. Strategies of arbuscular mycorrhizal fungi when infecting host plants. New Phytologist, v.130, p.3-21, 1995.

BOWEN, G.D. The biology and physiology in infection and its development. In: SAFIR, G.R. (Ed.). Ecophysiology of VA mycorrhizal plants. Boca Raton: CRC, 1987. p.27-55.
COSTA, M.C.B.; HAAG, H.P.; SARRUGE, J.R. Nutrição mineral de hortaliças. XIX. Absorção de macro e micronutrientes pela cultura do quiabeiro (Hibiscus esculentum L.). In: REUNIÃO DA SOCIEDADE BRASILEIRA DE HORTICULTURA, 29., 1972, Piracicaba. Anais. Piracicaba: ESALQ, 1972. p.109-125.

DANIELS, B.A.; SKIPPER, H.D. Methods for the recovery and quantitative estimation of propagules from soil. In: SCHENK, N.C. (Ed.). Methods and principles of mycorrhizal research. St. Paul: The American Phytopathological Society, 1982. p.29-35.

EVANS, D.G.; MILLER, M.H. Vesicular-arbuscular mycorrhizas and the soil-disturbance-induced reduction of nutrient absorption in maize. I. Causal relations. New Phytologist, Oxford, v.110, p.67-74, 1988.

HARLEY, J.L.; SMITH, S.E. Mycorrhizal symbiosis. London: Academic, 1983.483p.

HAYMAN, D.S.; DAY, J.M.; DYE, M. Preliminary observation on dual incoculation of white clover. In: GIANINAZZI-PEARSON, V.; GIANINAZZI, S. (Eds.). Physiological and genetical aspects of mycorrhizae. Dijon: INRA, 1986. p.467-473.

HEPPER, C.M. Techniques for studying the infection of plants by vesicular-arbuscular mycorrhizal fungi under axenic conditions. New Phytologist, Oxford, v.88, p.641-647, 1981.

JAKOBSEN, I. Vesicular-arbuscular mycorrhiza in fieldgrown crops. III. Micorrhizal infection and rates of phosphorus inflow in pea plants. New Phytologist, Oxford, v.104, n.4, p.573-581, 1986.

KRISHNA, K.R.; BAGYARAJ, D.J. Effect of vesiculararbuscular mycorrhiza and soulble phosphate on Abelmoschus esculentus (L.) Moench. Plant and Soil, Netherlands, v.64, p.209-213, 1982.

PACOVSKY, R.S.; FULLER, G. Development of two endomycorrhizal symbiosis on soybean in comparison with phosphorus fertilization. Plant and Soil, Netherlands, v.95, p.361-377, 1986.

PHILIPS, J.M.; HAYMAN, D.S. Improved procedure for clearing roots and staining parasitic and vesiculararbuscular mycorrhizal fungi for rapid assessment of infection. Transaction of the British Mycological Society, v.55, p.158-161, 1970.

PORTER, W.M. The most probable number method for enumerating infective propagule of vesicular- 
arbuscular mycorrhizal fungi in soil. Australian Journal of Agricultural Research, v.17, p.515$519,1979$.

ROSSIELLO, R.O.P.; ARAÚJO, A.P.; MANZATTO, C.V.; FERNANDES, M.S. Comparação dos métodos fotoelétrico e da interseção na determinação de área, comprimento e raio médio radicular. Pesquisa Agropecuária Brasileira, Brasília, v.30, n.5, p.633638, 1995.

SANDERS, F.E.; SHEIKH, N.A. The development of vesicular-arbuscular mycorrhizas infection in plantroot systems. Plant and Soil, Netherlands, v.71, p.223246, 1983.

SIEMONSA, J.S. La culture du gombo (Abelmoschus spp.) legume frut tropica. Wageningen: Landbouwhogeschool, 1982.277p.
SMITH, S.E.; GIANINAZZI-PEARSON, V. Physilogical interactions between symbionts in vesicular-arbuscular mycorrhizal plants. Annual Review of Plant Physiology and Plant Molecular Biology, v.39, p.221-224, 1988.

SMITH, S.E.; READ, D.J. (Eds.). Mycorrhizal symbiosis. London: Academic, 1997.605p.

SYLVIA, D.M. Activity of external hyphae of vesiculararbuscular mycorrhizal fungi. Soil Biology and Biochemistry, v.20, n.1, p.39-43, 1988.

TEDESCO, J.M. Extração simultânea de N, P, K, Ca, Mg em tecido de plantas por digestão por $\mathbf{H}_{2} \mathbf{O}_{2}-\mathbf{H}_{2} \mathbf{S O}_{4}$. Porto Alegre: UFRGS, 1982. 23p. (Informativo Interno, 1). 